\title{
Escape from Violence: What Reduces the Enduring Consequences of Adolescent Gang Affiliation?
}

\author{
Beidi Dong, Ph.D. \\ Department of Biostatistics and Epidemiology \\ University of Pennsylvania \\ Marvin D. Krohn, Ph.D. \\ Department of Sociology and Criminology \& Law \\ University of Florida
}

Beidi Dong is currently a postdoctoral fellow at the Penn Injury Science Center of the University of Pennsylvania. He received his Ph.D. in Criminology, Law and Society from the University of Florida. His primary research interests include developmental and life-course criminology, crime and place, and youth gangs and violence prevention. Marvin D. Krohn is a professor in the Department of Sociology and Criminology \& Law at the University of Florida. He is primarily interested in developmental approaches to the explanation of delinquency, drug use, and crime. He is a co-principal investigator of the Rochester Youth Development Study (RYDS), a three generational longitudinal panel study targeting those at high risk for serious crime and delinquency. He was elected a Fellow of the American Society of Criminology in 2012. Correspondence to: Beidi Dong, 936 Blockley Hall, University of Pennsylvania, 423 Guardian Drive, Philadelphia, PA 19104, United States. E-mail:

bdong@mail.med.upenn.edu 


\section{Introduction}

There is a considerable body of literature linking adolescent gang affiliation to both immediate and long-term negative developmental outcomes across a variety of life domains. Gangs function as a crime-facilitating context (Thornberry, Krohn, Lizotte, \& Chard-Wierschem, 1993), and the link between gang membership and delinquency, especially serious and violent offending, holds across time, place, demographic subgroups, data sources and definitions of gangs and gang membership (Klein \& Maxson, 2006; Krohn \& Thornberry, 2008). The link also holds up when gang members were compared with non-gang youth surrounded by highly delinquent friends (Battin, Hill, Abbott, Catalano, \& Hawkins, 1998; Huizinga, 1996; Thornberry, Krohn, Lizotte, Smith, \& Tobin, 2003). Importantly, the unique effects of gang membership are not confined to periods of active gang involvement (Melde \& Esbensen, 2011; 2014). Individuals with a history of gang affiliation are more likely to experience precocious transitions, have lower educational achievement, endure unemployment, suffer economic hardship and family problems in adulthood, and engage in sustained delinquency and exhibit an increased probability of arrest (Augustyn, Thornberry, \& Krohn, 2014; Krohn, Ward, Thornberry, Lizotte, \& Chu, 2011; Levitt \& Venkatesh, 2001a, 2001b; Pyrooz, 2014; Thornberry et al., 2003).

Despite evidence of the enduring consequences of gang affiliation, prior research has also suggested that not every gang youth experienced the negative consequences mentioned above (Moore, 1991; Sanchez-Jankowski, 1991). Surprisingly, there is little research on what reduces the long-term, negative impact of adolescent gang affiliation. We consider this an important question from both a theoretical and policy-making standpoint. Existing research on gang desistance has shown that declaring oneself a "former" gang member is not functionally the same 
thing as having no contacts with former gang associates (Decker \& Lauritsen, 2002; Pyrooz, Decker, \& Webb, 2014). Maintaining persisting social and emotional ties to a gang leads to perpetration and victimization after desistance (Carson \& Vecchio, 2015). It is therefore theoretically meaningful to comprehend what factors may buffer the continuing risk associated with gang joining on later offending. This is especially important when we recognize that gang membership is a relatively temporary phenomenon among a majority of youth who participate in a gang (Krohn \& Thornberry, 2008). Using a nationally representative sample of youth, Pyrooz and Sweeten (2015) recently estimated that approximately 401,000 juveniles join gangs and 378,000 exit gangs annually in the United States, with a turnover rate of $36 \%$. Although primary (and secondary) prevention remains the cornerstone of efforts to reduce the burden of violence and other gang-related problems (Simon, Ritter, \& Mahendra, 2013), the sheer number of exgang members calls for attention to tertiary prevention strategies that may alleviate the social and economic losses related to ex-gang members' sustained criminal involvement.

Against this background, the current study sets out to explore what factors moderate the relationship between adolescent gang affiliation and violence in established adulthood. To answer this question, we go beyond prior research in at least two ways. First, we take into account developmental heterogeneity in gang membership. Although gang scholars have called for a move beyond the dichotomy of "gang member" versus "non-gang member" and recognize the existence of developmental differences in gang affiliation (Maxson, 2013; Pyrooz, 2013), existing research has scarcely examined how distinct trajectories of gang affiliation may differentially affect long-term consequences of joining a gang. Second, we integrate Cullen's (1994) social support theory to examine possible insulators from the snares of gang affiliation (Moffitt, 1993). Past research has repeatedly shown that the failure of securing support from 
conventional sources is one of the most important reasons for gang involvement (Sharkey, Stifel, \& Mayworm, 2015). It follows that social support may also deflect the effects of gang membership on later violence. We thus test Cullen's propositions regarding social support and its protective effects against being a juvenile gang member.

\section{Adolescent Gang Affiliation and Long-Term Consequences}

Previous research has conceptualized adolescent gang affiliation as an "acute" turning point that may redirect normative trajectories in many life domains (Melde \& Esbensen, 2011; Sampson \& Laub, 2005). From a life-course perspective, attitudes and behaviors initiated during adolescence can have important consequences for successful transitions into adult roles and responsibilities (Elder, 1998). Specifically, gang researchers have documented the theoretical mechanisms that associate adolescent gang affiliation with subsequent life adversities. First, gang membership exerts criminogenic influences on individuals. Adolescent gang affiliation corresponds with increases in both law-violating behaviors and violent victimization, which in turn leads to tangible (e.g. arrest, gang enhancement penalties or incarceration) and intangible (e.g. labeling and stigmatization) consequences. Evidence has also shown that gang participation leads to long-lasting physical and mental health problems (Gilman, Hill, \& Hawkins, 2014; Wood, Foy, Layne, Pynoos, \& James, 2002).

Second, joining a gang sets adolescents apart from conventional society. Previous work has characterized the youth gang as a surrogate family (Vigil, 1988), a collective response to adverse social conditions (Hagedorn, 1998) and a group response to perceived threats (Klein, 1971; Moore, 1991). Decker and Van Winkle (1996) also reported that "involvement in legitimate social institutions or with non-gang peers and relatives drops dramatically following gang initiation" (p.187). Youth gangs have become the primary social network and reference 
group for gang members. Supervision and monitoring as well as bonding to conventional society are largely cut off. Meanwhile, gang involvement signifies a commitment to an alternative cultural orientation. Denied legitimate means to status, respect and acceptance pushes gang youth toward "focal concerns of lower class culture" such as toughness, smartness, autonomy or fate (Cloward \& Ohlin, 1960; Cohen, 1955; Miller, 1958). Gang experiences and orientation thus inhibit the acquisition of human and social capital from conventional social institutions (e.g. family, school or legitimate labor market) that is essential for later life success (Dong, Gibson, \& Krohn, 2015; Krohn et al., 2011).

Third and relatedly, gang affiliation leads to unstructured routine activities and deviant life styles. Gang joining exposes individuals to a street-oriented life style—-spending days and nights out on the streets with fellow members, drinking alcohol, smoking marijuana or hustling for drug sales. Taylor and colleagues (Taylor, Peterson, Esbensen, \& Freng, 2007; Taylor, Freng, Esbensen, \& Peterson, 2008) found that gang members are more likely than other youth to engage in unsupervised activities with peers and to hang out where drugs and/or alcohol are available. Such delinquent lifestyles and routine activities mediate the gang membership-serious violent victimization link. When life difficulties arise, ex-gang members often feel the temptation to return to their gang friends (and old life styles) for material and emotional support (Moloney, MacKenzie, Hunt, \& Joe-Laidler, 2009).

Finally, gang membership contributes to the formation of deviant self-concept and "anger identity" (Giordano, Schroeder, \& Cernkovich, 2007). Youth gangs develop "group esteem" in place of self-esteem, and in-group solidarity and intergroup conflict build upon and reinforce each other through self-sustaining cycles (Klein \& Maxson, 2006). The collective norms of youth gangs support and expect aggressive action when derogation of "collective honor" occurs, 
and individuals that identify with the gang are expected to quickly resort to violence above levels motivated by individual proclivities (Hennigan \& Spanovic, 2012; Papachristos, 2009). In addition, the development of "oppositional culture" and viewing oneself as a victim of oppression or the unfair target of racism and inequality further mobilizes techniques of neutralization among gang youth (Esbensen \& Deschenes, 1998; Moore \& Vigil, 1989).

Both ethnographic and quantitative empirical research suggest evidence for long-term negative life outcomes years after active gang participation. Sanchez-Jankowski (1991, p.61-62) discussed several possible outcomes of gang affiliation. Former gang members could 1) pursue various illegal economic activities on their own; 2) move on to another type of criminal organization or association, such as smaller groups like "crews" or branches of organized crime; 3) be incarcerated for a considerable part of their lives and perhaps involve themselves in the prison gangs; 4) die from drug overdose, violent confrontation or the risks of lower-class life or 5) pursue legitimate employment and maintain an ordinary lifestyle they avoided in the past. Moore (1991) identified three types of adult outcomes for ex-gang members: "squares", accounting for approximately 40 percent of the sample, were employed as young adults and lived a conventional family life, whereas "tecatos" (heroin addicts) and "cholos" (the undereducated and unemployed) accounted for one-quarter and one-third of the sample respectively. For the latter two categories, the gang remained the first place they turned to when encountering life difficulties. Hagedorn (1998) found that only a third of male gang members in his study had a high school diploma and about the same number were employed. The rate for high school graduation was equally low for female gang members. Nearly 9 of 10 female gang members were mothers by their early $20 \mathrm{~s}$, with about $60 \%$ on welfare. 
While ethnographic studies have presented us with detailed descriptions of ex-gang members' lives, quantitative studies with a longitudinal design better control for confounding factors that might lead to adulthood adversity such as self-selection mechanisms, memory recall, or other unobserved factors rather than gang affiliation itself. Levitt and Venkatesh (2001a, 2001b) found that once background characteristics such as GPA and family environment were controlled, earlier gang affiliation remained positively associated with ever having been incarcerated, number of times shot, and annual illegal income, and negatively associated with annual legal income.

The Rochester Youth Development Study provided some of the most important findings regarding the long-term consequences of gang membership. Thornberry et al. (2003) found that male gang members who stayed in a gang for more than one year (i.e. stable gang members) were more likely to drop out of school, impregnate a girl, be a teenage parent, experience unstable employment, and cohabitate than non-gang members after controlling for background variables. For females, gang membership increased the odds of early pregnancy, teenage parenthood and unstable employment after controlling for selection variables. Additionally, adolescent gang affiliation had both a direct and an indirect effect, through the impact on precocious transitions, on the probability of arrest at 20-23 years of age.

Krohn et al. (2011) extended the previous study by exploring even longer-term effects of adolescent gang affiliation. Their analysis covered a 17-year period from when the subjects were approximately 14 years old until about 31 . They found that male subjects with longer periods of gang affiliation in adolescence were more likely to experience precocious transitions, which in turn led to economic hardship and family problems. The unsuccessful fulfillment of adult roles and statuses eventually contributed to street crime as well as arrest in adulthood. Augustyn, 
Thornberry and Krohn (2014) found that gang affiliation increased the likelihood of maltreatment perpetration; precocious life transitions mediated the association between gang membership and child maltreatment. These findings highlight the role of gang membership in the facilitation of "fragile families" (McLanahan, 2009).

Moreover, Pyrooz (2014) focused explicitly on the longitudinal effects of gang joining on educational attainment over a 12-year span. Through adjusting for nonrandom selection into gangs with propensity score matching technique, he found that on average gang avoiders completed 12.2 years of education versus gang joiners' 11.6 years of education. With regard to educational milestones, gang avoiders were more likely to obtain a high school diploma and to graduate from a four-year college. Using the same analytic technique, Gilman et al. (2014) reported that adolescent gang affiliation led to poorer outcomes in multiple areas of adult functioning, including higher rates of self-reported crime, receipt of illegal income, incarceration, drug abuse or dependence, poor general health, and welfare receipt and lower rates of high school graduation.

\section{Social Support and the Snares of Gang Affiliation}

An important, but largely unexplored, issue is what buffers the long-term negative impact of gang affiliation. Gang desistance literature suggests that leaving the gang is more of a process, and simply de-identifying is not enough. Major obstacles in the desistance process include emotional or social ties to the gang and maintained routine activities due to external factors such as attending the same school, residing in the same neighborhood as gang friends or the fact that some of these same peers may be relatives or were friends prior to joining the gang (Carson \& Vecchio, 2015; Pyrooz \& Decker, 2011). In fact, the process of leaving the gang has been characterized as being more difficult than joining, in part because the gang provides a source of 
support (Vigil, 1988) ${ }^{1}$. Gang members often choose not to completely leave the gang until a suitable substitute has been found.

Discovering protective factors will enable us to suggest ways in which to reduce the persisting ties maintained by ex-gang members to a gang and perhaps, provide alternative sources of social support for ex-gang members when difficulties arise. It should be recognized that additional labels and formal controls may backfire, merely reinforcing gang cohesiveness and dependence (Klein \& Maxson, 2006). "Efforts to lessen the pernicious and continuing consequences of gang membership will involve the knifing off of gang ties and stigma, identity and cognitive transformation, and repairing social capital along with an infusion of prosocial structures and influences” (Pyrooz, 2014, p.76; see also Decker, Pyrooz, \& Moule, 2014). Cullen's (1994) social support theory provides us with an appropriate framework to address how members may avoid the snares of gang affiliation.

Cullen (1994) integrated for the first time the diverse insights on social support into a coherent criminological paradigm. Lin (1986) defined social support as "the perceived or actual instrumental and/or expressive provisions supplied by the community, social networks, and confiding partners" (p.18). Implied in this definition are three key dimensions of support. First, social support can be perceived or actual. Prior research has shown that the effects of perceived support are stronger and consistently beneficial (Thoits, 2011; Uchino, 2009). Second, social support can be instrumental (providing advice/guidance as well as financial or material resources) or expressive (satisfying individuals' need for love and affection, esteem and identity, and belonging and companionship). Third, social support exists at different levels of society and comes from distinct sources. Social support from conformist sources (e.g. parents) is most likely

\footnotetext{
${ }^{1}$ Fear of consequences acts as a barrier in the disengagement process. Other negative consequences of gang desistance include still being viewed as gang-involved by rival gang members, police, and people in the community, experiencing threats or losing gang friends (Carson, Peterson, \& Esbensen, 2013; Decker \& Lauritsen, 2002).
} 
to combat criminogenic influences, whereas illegitimate support networks (e.g. deviant friends) assist individuals with a sense of belonging, role models, skills and resources that, unfortunately, contribute to an accumulation of "criminal capital" (Hagan \& McCarthy, 1997). Moreover, social support can be delivered in a more or less consistent manner. The consistent provision of social support creates a sense of trust between the recipient and the provider(s) of support, which "forms the basis for a strong social bond that becomes generalized into a strong moral commitment to others and to legitimate social institutions" (Colvin, Cullen, \& Vander Ven, 2002, p.24).

Besides understanding social support as a theoretical concept, it is necessary to explicate the mechanisms through which networks of social support may lessen the effects of gang membership on subsequent offending. Cullen's (1994) propositions on social support and crime can be rephrased into three main mechanisms: 1) networks of social support create a nurturing environment that provides acceptance and a sense of belonging, and affirms one's self-worth and dignity. Within a supportive network, people will signal empathy and acceptance rather than labeling and stigmatization for the individual in adverse situations, listen to his or her reactions and worries, accompany the individual as the stressful event unfolds, and help work out potential solutions (Clark, 1987; Shott, 1979). Perception of support reassures the individual's sense of truly "mattering" to others and sustains his or her sense of self-worth (Rosenberg \& McCullough, 1981). The reciprocal nature of supportive networks also induces voluntary or involuntary role obligations on the recipients, facilitating the development of a moral commitment and social bonds to legitimate social institutions.

2) Networks of social support supply physical and human capital needed to refrain from persistent offending and enhance prosocial learning. Compared to minority youth whose access 
to support was structurally limited, white juveniles from working-class neighborhoods were able to enter the legitimate workforce through family ties. When in trouble, they sought professional advice and aid from relatives on the police force or in the courts to escape being captured in a criminal role (Sullivan, 1989). The provision of social support also creates opportunities for prosocial learning. Perception of support allows people in despair to envision themselves in the future, which attenuates anxiety, bitterness and anger, and generates hope (Anderson, 1999; Maruna, 2001). Meanwhile, people may learn to deliver support to others, which can "transform selves, inculcate idealism, foster moral purpose, and create longstanding interconnections - all of which would seem anti-criminogenic" (Cullen, 1994, p.543).

3) Social support serves as a precondition for effective formal and informal social control. Colvin et al. (2002) argued that social support and control are not rival concepts; "normative control can involve the delivery of expressive social support and remunerative control can involve the delivery of instrumental social support" (p.26). This is particularly important when handling gang youth who commit to an "oppositional culture" and feel antagonistic and resistant to conventional authority. There is ample evidence that control is most effective when it is exerted as part of a supportive rather than a detached or punitive relationship (e.g. Braithwaite, 1989; Sherman, 1993; Wright \& Cullen, 2001).

A handful of studies have shown that perception of support, in particular parental support, moderated the relationship between associating with deviant friends and delinquency and drug use. For instance, Poole and Regoli (1979) observed that delinquent friends made a greater difference in delinquency for adolescents who had weak family support than those who had strong family support. Frauenglass, Routh, Pantin, and Mason (1997) found that family social support reduced the influence of deviant peers on adolescents' tobacco and marijuana use 
in spite of insignificant main effects. Marshal and Chassin (2000) reported gender-specific effects of family support on the association between adolescents' affiliation with drug-use promoting peers and subsequent alcohol use. For girls, both maternal and paternal social support buffered the effect of peer influence on alcohol use, whereas, for boys, maternal social support strengthened the association and paternal support did not moderate peer influence. Allen, Chango, Szwedo, Schad, and Marston (2012) also found that baseline peer substance use was more likely to predict relative changes in substance use over the following year for adolescents who had experienced less maternal support.

Parental attachment can be considered an alternative measure of parental social support. If youth have strong affective bonds to their parents, it is likely that parents provide at least the type of expressive support mentioned above. Studies have found that parental attachment is another important moderator on the relationship between negative peer influence and later delinquency and drug use. For example, Vitaro, Brendgen, and Tremblay (2000) observed that boys' attachment to parents moderated the link between best friend's deviancy and their own later delinquency. Dorius, Bahr, Hoffmann, and Harmon (2004) also reported that closeness to a parent moderated the relationship between peer drug use and adolescent marijuana use.

Given that youth gangs are qualitatively different from non-gang delinquent peer groups in important aspects (Dong \& Krohn, 2016), conclusions drawn from the aforementioned studies might not be applicable to gang joiners. Yet, few studies have examined the protective effects of parental support on the relationship between gang affiliation and subsequent offending. WalkerBarnes and Mason (2004) reported findings that are most relevant to the current study. Using an ethnically diverse sample of 300 ninth grade students in Miami, they found that parental behavioral and psychological control offset the relationship between gang involvement and 
adolescent problem behavior, whereas parental warmth unexpectedly strengthened that relationship. Although their study represented an important first step in identifying factors that may strengthen or attenuate the association between gang involvement and juvenile delinquency and substance use, Walker-Barnes and Mason (2004) were not able to explore what offsets the lingering effects of gang membership years after leaving the gang.

In addition to parental social support, acquiring a stable partner relationship may provide another source of social support. Sampson and Laub (1993; Laub \& Sampson, 2003) demonstrated that having a satisfying relationship within a marriage deflected crime trajectories among male offenders. Warr (1998) noted that the decline of delinquency following transitions to marriage and family was largely introduced by changes in peer relations, particularly time spent with peers. It follows that becoming involved in a partnership decreases gang members' interactions with former accomplices. Other studies have also found support for the protective effects of a good marriage or stable partner relationship (e.g. Osgood \& Lee, 1993; Sampson, Laub, \& Wimer, 2006).

\section{The Current Study}

Since youth gangs can be viewed as "prototypical deviant social networks whose actors are embedded in a culture and behavior system that both facilitates deviant behavior and isolates the individuals from prosocial networks" (Thornberry et al., 2003, p.166), it is not surprising to observe the long-term negative outcomes of adolescent gang affiliation. The present study aims to investigate what buffers the continuing risk associated with gang joining many years after leaving the gang. We hypothesize that parental social support will protect former gang members from continuing violent behavior. We also anticipate that becoming involved in a partner relationship will reduce the impact of gang membership on subsequent violence. We further 
hypothesize that different protective factors will exist for individuals in distinct trajectories of gang affiliation.

Krohn and Thornberry (2008) suggested that younger teenagers are more apt to join a gang due to the influence of a friend or older family member, whereas those who join at an older age may be seeking protection or profit from their membership. Joining a gang at a younger age may be more transitory in nature and as youth become more mature, they may realize that gang membership is not in their interest. On the other hand, gang participation in later teenage years may represent a more deliberate choice on the youth's part that involves greater commitment to the gang and the behaviors that are part of gang life. Decker and Curry (2000), for instance, found that gang involvement is not a "master status" among younger adolescents but means more for older teenagers and individuals in their $20 \mathrm{~s}$. Thus, for early-adolescence gang members who are more malleable, a life event such as establishing a partnership that may re-structure their daily routines and reduce the influence of gang friends may be most helpful, whereas, for lateadolescence gang members, perception of consistent support from conformist sources is needed to buffer against the continuing "attractions" and criminogenic influences associated with earlier gang affiliation.

\section{Methods}

\section{Data and Sample}

The data for the current study come from the Rochester Youth Development Study (RYDS), a longitudinal panel study aimed at understanding the development and consequences of serious delinquency and drug use. Data collection began in 1988 with a high-risk sample of 1000 seventh- and eighth-grade students in the public schools of Rochester, New York. Due to the relatively low base rates for serious and chronic delinquency, the sample was stratified on 
two dimensions to provide high-risk respondents. First, males were oversampled ( $75 \%$ vs. $25 \%$ ) because they are more likely than females to engage in serious forms of delinquency and drug use. Second, students residing in high crime neighborhoods were oversampled assuming that living in such areas of the city represented enhanced risk for delinquency. To identify high crime neighborhoods, each census tract in Rochester was assigned a resident arrest rate reflecting the proportion of the total population living in that tract that was arrested by the Rochester police in 1986. Subjects were oversampled proportionate to the rate of offenders living in a tract. The sample was predominantly comprised of minorities (68\% African American, 17\% Hispanic, and $15 \%$ White) and males (73\%).

A total of 14 waves of data have been collected during the course of three phases of data collection, covering the subjects from their early teenage years (about age of 14) through age 31 . The data used in this study span all three phases of data collection. Specifically, Phase 1 covered the adolescent years of the subjects from about 14 to 18 years of age. In Phase 1, the respondents and their primary caretakers (most often biological mothers) were interviewed nine and eight times respectively at 6-month intervals (Wave 1-9). Phase 2 began after a 2.5-year gap in data collection. The respondents with their primary caretakers were interviewed at three annual intervals at ages 21 to 23 (Wave 10-12). Phase 3 consisted of respondent interviews at 29 and 31 years of age (Wave 13 and 14). Official data were also collected from schools, social services and the police. The attrition rate in the RYDS data has been acceptable. During Phase 3, over $76 \%$ of the original sample had been retained. An examination of the effects of attrition through Phase 3 indicates that attrition does not create significant bias in the key variables used in the analysis. 
The current investigation is limited to male subjects only because the prevalence of female gang membership was very low after the early adolescent years (Thornberry et al., 2003). We used data from Phase 1 to estimate developmental trajectories of gang affiliation during adolescence. Potential direct and buffering protective factors during emerging adulthood were examined using data from Phase 2 (Losel \& Farrington, 2012), and the outcome variable of interest—violence was assessed using data from Phase 3. To control for offending opportunity, individuals who were incarcerated during Phase 3 were excluded from the final analysis ${ }^{2}$.

\section{Measures}

Table 1 reports descriptive statistics for the key variables used in the study. The last column in the table specifies the waves from which the measures were taken.

Gang affiliation. Operational definitions of gang affiliation vary from more complex measures using gang names, symbols, or some notion of organizational structure to simpler measures relying on self-nomination (Curry, 2000; Thornberry et al., 2003). Previous research has found that the single self-reported question asking the respondents whether they were in a gang is a valid indicator of gang membership (Decker, Pyrooz, Sweeten, \& Moule, 2014; Esbensen, Winfree, He, \& Taylor, 2001). Beginning at Wave 2, the study subjects were asked whether they were members of a "street gang or posse" (the term used by Rochester adolescents at that time) since the date of the last interview. Thornberry et al. (1993) found that, in the RYDS, this single self-reported question results in an almost identical list of gang members as measures based on additional selection criteria, such as the size or name of the gang. Thus, at each wave of Phase 1 data collection, we used a dichotomous variable to indicate gang affiliation that was constructed using self-reported answers. Slightly more than 30\% of the study subjects

\footnotetext{
${ }^{2}$ As a robustness check, we also ran analyses when the incarcerated respondents were included. The same substantive findings were observed.
} 
were gang members at some point during adolescence, and very few respondents (fewer than 2\%) reported gang involvement after Wave 9.

Violence. We created a variety scale of violence to capture individuals' tendency to commit violent acts during adulthood. Sweeten (2012) suggested that variety scales are more "attractive" than dichotomous and frequency scales because "they are less sensitive to high frequency items, much less skewed than frequency scales, and have the highest concurrent validity and equal predictive validity to other scales" (p.553). Given that violent behavior is relatively rare among adults, we used a cumulative score combining data from Waves 13 and 14 to provide sufficient variation. The subjects responded to 5 questions about violent interactions with others including "attacked someone with a weapon", "hit someone with the idea of hurting them", "involved in gang or posse fights", "threw objects such as rocks or bottles at people", and "used weapons or force to make someone give you money or things". If they answered "yes" to a question at either Wave 13 or Wave 14, we considered that they had committed that violent act during adulthood and coded it as "1"; if they did not commit the offense at either wave, we coded it as " 0 ". Hence, the theoretical range of this variable is 0 to 5 . Table 1 shows that none of the respondents committed more than two of these violent acts ${ }^{3}$.

Consistency of parental support. Results from exploratory factor analyses suggest that perceived parental support during emerging adulthood is best captured by a one-dimensional measure. The respondents were asked 8 questions tapping how likely primary caregivers, for example, talk to you about problems, help you with important decisions, go places or do things with you, give or loan you money, and help in an emergency. Responses were scored "very unlikely" (1), "unlikely" (2), "likely" (3) and "very likely" (4), and items were averaged to

\footnotetext{
${ }^{3}$ As a robustness check, we also created a frequency score of violence. The same substantive findings were observed.
} 
provide the mean score ${ }^{4}$. As discussed earlier, the consistency of parental support should be more important than perceived support from any single wave. We thus dichotomized the score of perceived parental support at each of the three waves (Waves 10,11 and 12) during emerging adulthood when respondents were approximately 21 to 23 years old and created a new mean score on how many waves they scored in the top half $f^{5}$. To avoid nonessential multicollinearity and facilitate interpretation, we standardized the consistency score before estimating moderating effects (Dalal \& Zickar, 2012).

Consistency of parental attachment. Attachment to a parent figure is measured by an 11item scale, assessing warmth, closeness and lack of hostility in the parent-child relationship. The respondents were asked, for instance, how often you would say that "you get along with your primary caregiver", "you feel that you can really trust your primary caregiver" or "you really enjoy your primary caregiver". Responses were indicated on a 4-point scale from "never" (1), "seldom" (2), "sometimes" (3) to "often" (4), and items were averaged to provide the mean score $^{6}$. Following the same procedure described above, we created the standardized measure of consistency of parental attachment during emerging adulthood. The correlation between consistency of parental support and attachment during emerging adulthood equals 0.36 .

Partner status. Being in a stable partnership may be important in re-structuring individuals' routine activities. At each wave during emerging adulthood, individuals who were married, living with a partner (i.e. cohabitation) or in a relationship for at least 6 months were coded as "1", otherwise " 0 ". We used this binary indicator of partner status rather than examining partner support or satisfaction because approximately half of the respondents reported

\footnotetext{
${ }^{4}$ The Cronbach's alpha equals, respectively, 0.91, 0.89 and 0.92 at Wave 10, 11 and 12.

${ }^{5}$ A respondent needed to have provided at least two waves of data (out of three) to be included in the final analysis. A sum score was not used because it would not allow missing information on any wave of data.

${ }^{6}$ The Cronbach's alpha equals, respectively, 0.87, 0.86 and 0.88 at Wave 10, 11 and 12.
} 
not having a stable partner at any given wave during emerging adulthood. Similarly, we created a consistency score of partner status across emerging adulthood.

Control variables. We included several variables measured in early adolescence (Wave 2 or earlier) before most youth joined gangs in order to control for background characteristics. Dichotomous indicators were used to represent African-American and Hispanic race/ethnicity (reference group is white). Neighborhood concentrated disadvantage is measured by a 4-item latent measure constructed from the 1990 U.S. Census assessing the level of economic hardship within the neighborhood where the subjects lived. Standard census items included percentage in poverty, percentage female-headed households, percentage unemployed, and percentage receiving public assistance. Parental education refers to the highest grade completed by the principal family wage-earner. Family poverty is an indicator of whether a household had an income below the federally defined poverty level for a given family size when the respondents were on average 14.5 years of age (Wave 2). Academic aptitude is measured by the math percentile score received on the California Achievement Test in 1987 (when G2 subjects were approximately 12 years old). Higher scores on this variable indicate greater academic aptitude. Low parental supervision has been found of primary importance (among family characteristics) in predicting adolescent gang affiliation and delinquency (Klein \& Maxson, 2006; Thornberry \& Krohn, 2008). Parental supervision is measured by a 4 -item scale at Wave 2 regarding how often the primary caregiver knows where the respondent is and with whom and how important that is to the primary caregiver.

We also controlled for three important individual characteristics in the final analysis. Self-esteem is measured by a 9-item scale derived from Rosenberg's (1965) self-esteem scale at Wave 2. The subjects were asked to what extent they agree or disagree with a series of 
statements about oneself. Depression is measured by a 14-item scale tapping the frequency of depressive symptoms at Wave 2. Aggression is measured by a trimmed version of the aggression subscale of the Child Behavior Checklist, which was first administered to the primary caregivers during Wave 3. Primary caregivers were asked 12 questions about how often (always, sometimes, or never) the adolescent exhibited behaviors such as being restless and getting into fights. Finally, we controlled for prior violence at Wave 2.

\section{Data Analysis}

To answer our research questions, data analysis proceeded in three main steps using Stata (Version 14.0; StataCorp 1996-2015). First, developmental trajectories of adolescent gang affiliation were estimated. Following Nagin's (2005) two-stage model selection process, we first chose the optimal number of groups to include in the model mainly based on the Bayesian Information Criterion (BIC). In the second stage, the model was refined to determine the preferred order of the polynomial specifying the within-individual change for each trajectory given the first-stage decision on number of groups. Second, developmental trajectories of gang affiliation were linked to later violence through Poisson regression models ${ }^{7}$, testing whether gang involvement in adolescence put youth at heightened risk of violence after more than 10 years. Third, main effects and moderating effects models were estimated to establish the relationship between adolescent gang affiliation, direct and buffering protective factors during emerging adulthood, and violence in adulthood. To perform the regression analyses, we relied on the posterior probabilities of group membership from the best-fitting trajectory model. This approach takes into account classification uncertainties associated with the maximum probability assignment rule (Raudenbush, 2005). The data were screened for patterns of missingness, and we

\footnotetext{
${ }^{7}$ Since there is no significant evidence of overdispersion, the Poisson regression model with robust standard errors is preferred to the negative binomial regression model (Cameron \& Trivedi, 2009).
} 
found little evidence that the assumption of "missing at random" was violated. We thus

employed the technique of multiple imputation (mi impute chained; number of imputations $=20$ )

to deal with missing data in the present study (Allison, 2002) ${ }^{8}$.

\section{Results}

\section{Trajectories of Adolescent Gang Affiliation}

For gang affiliation during adolescence, we found a 3-group model, defined by one group following a zero-order trajectory and two groups following a quadratic trajectory, as the best fitting model for the current investigation (Figure 1) ${ }^{9}$. This solution has the best BIC score and acceptable values of minimum average posterior probability of assignment $(0.83)$ and the odds of correct classification (6.23). Not surprisingly, the majority of the respondents (76.5\%) demonstrate very low probabilities of gang affiliation during their adolescent years. The "early adolescence" group (15.3\%) exhibits relatively high probabilities of gang affiliation that drop off after age 14. On the contrary, the "late adolescence" group (8.2\%) has low probabilities of gang affiliation during early adolescence. These probabilities rise to a peak around age 17 and decline gradually after that. Using a comparable sample from the Montreal Longitudinal-Experimental Study, Lacourse et al. (2003) identified almost identical trajectories of male gang membership during adolescence.

\section{Reducing the Long-Term Impact of Gang Involvement}

Consistent with prior research, adolescent gang affiliation predicts violence in established adulthood. Table 2 shows that compared with individuals in the "very low" trajectory,

\footnotetext{
${ }^{8}$ We excluded subjects from Poisson regression analysis if they had missing information on the outcome variable, although violence in adulthood was included in the imputation model. We observed no statistically significant differences between the analysis sample $(N=560)$ and those not included in the analysis with respect to all included variables, except that those not included in the analysis sample experienced lower levels of depression.

${ }^{9}$ To guard against local solutions in the estimation of growth mixture models, we estimated trajectory models using multiple sets of starting values (Hipp \& Bauer, 2006).
} 
individuals in both trajectories of active gang involvement exhibit significantly higher levels of violence in adulthood. The incidence rate ratios (IRR) of 2.587 and 2.986 suggest that such lingering effects are substantial. While holding all other variables in the model constant, being in the early- and late-adolescence trajectory groups leads to an increase in the subject's incidence rate for violence by $158.7 \%$ and $198.6 \%$, respectively.

Model 1 in Table 3 reports Poisson regression results of violence on gang affiliation trajectories and partner status at Wave 10. Developmental trajectories of gang affiliation remain significant predictors of violence after taking into account partner status at Wave 10. Having a partner at Wave 10 leads to a higher level of violence among the overall sample (IRR=1.677, $p=0.039$ ), indicating potential mate selection effects for the high-risk RYDS subjects.

Interestingly, results from the moderating effects model (Model 2 in Table 3) suggest buffering protective effects of having a partner at Wave 10 for "early-adolescence" gang members (IRR=0.194, $p=0.015)$. Compared with individuals in the "very low" trajectory, "earlyadolescence" gang members without a partner at Wave 10 have an incidence rate for violence that equals 6.496; this number equals 1.260 for "early-adolescence" gang members with a partner. It appears that having a partner relatively early in life protects "early-adolescence" gang members from violence in adulthood despite the fact that the partner might be delinquency-prone as well. In analyses not shown here, we also investigated the potential protective effects of having a partner at later waves and the consistency of having a partner across emerging adulthood. Significant moderating effects were not observed. Thus, the early timing of having a potentially life-changing event is important when averting early joiners from the sustained influence of adolescent gang affiliation. 
Model 1 in Table 4 reports the results from Poisson regression in which violence is regressed on gang affiliation trajectories, consistency of parental support and attachment, and control variables. Gang affiliation trajectories remain significant predictors of violence after taking into account consistency of parental support and attachment across emerging adulthood. Consistency of parental attachment manifests main or direct protective effects (IRR $=0.695$, $p=0.016)$ on violence.

Model 2 in Table 4 shows the results from the moderating effects model that includes the interactions between gang affiliation trajectories and consistency of parental support. Although consistency of parental support does not exhibit main or direct protective effects, it buffers against risk of later violence for late-adolescence gang members (IRR $=0.361, p=0.016)$. The magnitude of such buffering protective effects is substantial. "Late-adolescence" gang members with consistency of parental support that is one standard deviation below the average have an incidence rate for violence 5.875 times that of individuals in the "very-low" trajectory; this number equals 2.121 for "late-adolescence" gang members with an average level of consistency of parental support.

Model 3 in Table 4 reports the results from the moderating effects model including the interactions between gang affiliation trajectories and consistency of parental attachment. Despite the significant main or direct protective effects, consistency of parental attachment dose not exhibit significant buffering protective effects against risk of later violence for either group of gang youth. In analyses not shown here, we combined Model 2 and Model 3 in Table 4 into one model; the same substantive results were observed. To facilitate interpretation, we presented 
separate models ${ }^{10}$. In brief, the results are consistent with our hypothesis that different protective factors are needed for individuals following distinct trajectories of gang affiliation, indicating the importance of studying developmental heterogeneity among gang members ${ }^{11}$.

\section{Discussion}

While it is important to recognize the problematic consequences of adolescent gang membership on the life course of those who at one time were members of those organizations, we must also acknowledge that not all former gang members experience them. This observation raises the question of what distinguishes those who are adversely affected by their gang experience from those who are not.

We conceptualized gang affiliation as a risk factor for future violent behavior and asked the question of what protective factors might insulate former gang members from the negative consequences of having been a member of a youth gang. Past research has suggested that one of the essential roles that gangs play in the lives of their members is to provide social support (Hagedorn, 1998; Moore, 1991; Vigil, 1988). We hypothesized that if gang members continued to receive social support from more conventional sources such as their parents or stable partners, the impact of the gang on their long-term life chances and violent criminality would be lessened and they would be less likely to experience problematic outcomes.

Cullen (1994) explicitly based his theory on the importance of social support. He suggested that social support could exhibit both main effects of crime reduction and moderating effects of criminogenic risk buffering. To examine both the direct and buffering protective

\footnotetext{
${ }^{10}$ To further check the robustness of our findings, we also investigated the interactions between gang affiliation trajectories and partner status at Wave 10, consistency of parental support and attachment in the same model. The same substantive results were observed.

${ }^{11}$ In analyses not shown here, we also examined the main and moderating effects of consistency of peer support on the relationship between gang membership trajectories and violence in adulthood. Statistically significant results were not observed. This might be due to the high-risk nature of the sample; the RYDS data cannot differentiate between conventional and delinquent peer support.
} 
effects of social support, we used three measures, consistency of parental social support, consistency of parental attachment, and the establishment of a stable partner relationship. We hypothesized that these measures of social support would have different effects on the distinct trajectories of gang membership.

Trajectory group analysis distinguished gang members who entered the gang early in adolescence and left the gang from those who joined the gang in later adolescence and had a presumably more sustained membership. Different measures of social support interacted with these two trajectory groups differently to protect them against continued violent behavior. Consistency of parental attachment did not have a moderating effect on violent behavior for either trajectory group although it did have an overall main or direct effect on violence. Contrastingly, consistency of parental social support did not have a main effect but did interact with the late-adolescence trajectory group to moderate the impact of gang affiliation. Having a partner relatively early in life served to protect those individuals in the early-adolescence trajectory group from sustained violence.

The interactions for the two trajectory groups suggest that the decision to take into account the pattern of gang membership over time was important. The findings regarding partner status are complex. While the main effect of partner status is positively related to later violence, the interaction term between partner status and the early-adolescence trajectory group indicates that for that group having a partner relatively early in life decreases the probability of violent behavior. Prior research on early sexual activity and early co-habitation indicates that precocious partnering is co-morbid with criminal behavior. The main effect observed in our results confirms this observation. However, once in a stable partner relationship, the relationship reduces the impact of gang membership on violent crime but only for the early gang joiners. Partner status 
does not protect the late joiners from violence. We suspect that this is because the earlyadolescence group is involved in the gang in a more experimental way and the social support offered in an early partner relationship is sufficient to moderate the more problematic consequences of gang membership represented by violent crime. On the other hand, the lateadolescence trajectory may represent a more committed group of gang members. Their reasons for joining a gang may be the consequence of more serious economic and family concerns. For this group, early entry into a partner relationship operates in the more characteristic manner of being co-morbid with criminal behavior. Future research needs to examine the different reasons for gang joining for these two groups and the possibility that the partner relationships differ in terms of their quality and the age at which they occurred.

We had anticipated that parental support would moderate the relationship between violent crime and gang membership for both trajectory groups. However, our results indicate that it does so only for the late-adolescence trajectory group. For this group, having parents who provide a combination of instrumental and expressive social support diminishes the impact of having been in a gang on problematic consequences. If our assumption about the greater commitment to the gang of individuals in this group is correct, the findings suggest a promising avenue of preventing violent crime even among those most likely to commit it. Parental social support may provide the type of emotional needs that individuals who have experienced the potentially turbulent disruptions in the family that often accompany adolescence need in order to reestablish the bond with their family. Additionally, if the gang provided a means of instrumental support for youth who were members of a gang during adolescence, the opportunity to fall back on that type of support from parents may be essential if youth are to disaffiliate with a criminal lifestyle. Again, confirming these speculations is essential in determining the basis for our findings. 
Future research may further explore the role of parental social support and attachment for early gang joiners.

The current study is subject to several limitations. First, the findings were derived from a high-risk, predominantly African American sample in Rochester, NY, a new or "emergent" gang city $^{12}$. Gang composition, structure, activities and residents' reactions to gangs differ in emergent versus traditional gang cities (Curry, 2000; Howell, 2012; Klein, 1996). It is not clear to what extent our findings can be replicated in other contexts, especially traditional gang cities like Chicago or Los Angeles. Second, the present findings are limited to males only. Although prior research has identified similarities in risk factors and delinquency facilitating effects between male and female gang members, important differences still exist (Miller, 2001; Moore, 1991). With appropriate data, future research should validate our conclusions with a female sample. Third, because approximately half of the respondents reported not having a partner at any given wave during emerging adulthood, we assessed the protective effects of partner status rather than partner support or satisfaction. Finally, developmental trajectories of self-reported gang affiliation are not necessarily reflecting varying levels of gang commitment. Self-identification as a gang member is a manifestation of the latent trait of "gang embeddedness", a new concept that captures individual immersion in a gang (Pyrooz, Sweeten, \& Piquero, 2013). A future member may increase his/her level of embeddedness in a gang prior to joining; likewise, a former member is likely to have residual or non-zero levels of gang embeddedness after leaving.

\section{Conclusions}

Disengaging from a gang is a process in which individuals typically become disillusioned with gang life or experience feelings of maturation. Ex-gang members begin to explore

\footnotetext{
12 Thornberry et al. (2003) reported that gangs emerged in Rochester in the mid-1980s; the Rochester Police Department reported 1985 as the year of onset to the National Youth Gang Center.
} 
opportunities external to gang life and typically drift away from their gang friends (Carson \& Vecchio, 2015). Decker, Pyrooz and Moule (2014) reported that increased family ties were important in the disengagement process; our research suggests that continuing family ties through emerging adulthood are also vital for lessening the impact of gang membership on subsequent crime. Perception of social support from conventional sources (e.g. parents or a stable partner) may facilitate the process and reduce the long-term, negative impact of adolescent gang affiliation.

More broadly, there are two points of emphasis that are suggested by the findings reported here. First, social support needs to be taken into consideration when examining why those who experience such important risk factors for violent crime such as gang membership, are less likely to continue to commit such behavior. Cullen (1994) effectively outlined the reasons for why theories and research on crime should consider social support as a critical variable in the etiology of criminal behavior and as a protective factor in the face of risk. Our findings lead us to concur with Cullen's assessment and his call for "good criminology" (Cullen, 1994, p.552), challenging the current hegemony of punitive policy in criminal justice. Public policies should aim toward providing a consistent level of social support to high-risk adolescents and their families over time. Importantly, these support efforts must be non-punitive, acceptable to families and sensitive to the contexts in which they are offered (Shute, 2013).

Second, the fact that results varied for the two trajectory groups is important for both future research and practice. As suggested above, future research needs to explore in more detail the possible explanations for the differences we observed. However, the findings contribute to a growing body of research that suggests that the developmental pattern of behavior (in this case gang membership) may be as important, if not more so, than either the prevalence or incidence of 
that behavior. Accordingly, not differentiating sub-categories of gang members in a developmental sense limits our capability to steer subjects toward the most appropriate intervention and remediation programs. 


\section{References}

Allen, J. P., Chango, J., Szwedo, D., Schad, M., \& Marston, E. (2012). Predictors of susceptibility to peer influence regarding substance use in adolescence. Child Development, 83, 337-350.

Allison, P. D. (2002). Missing data: Quantitative applications in the social sciences. Thousand Oaks, CA: Sage.

Anderson, E. (1999). Code of the street: Decency, violence, and the moral life of the inner city. New York, NY: Norton.

Augustyn, M. B., Thornberry, T. P., \& Krohn, M. D. (2014). Gang membership and pathways to maladaptive parenting. Journal of Research on Adolescence, 24, 252-267.

Battin, S. R., Hill, K. G., Abbott, R. D., Catalano, R. F., \& Hawkins, J. D. (1998). The contribution of gang membership to delinquency beyond delinquent friends. Criminology, 36, 93-115.

Braithwaite, J. (1989). Crime, shame, and reintegration. Cambridge, MA: Cambridge University Press.

Cameron, A. C., \& Trivedi, P. K. (2010). Microeconometrics using Stata. College Station, Texas: Stata Press.

Carson, D. C., Peterson, D., \& Esbensen, F. A. (2013). Youth gang desistance: An examination of the effect of different operational definitions of desistance on the motivations, methods, and consequences associated with leaving the gang. Criminal Justice Review, $38,510-534$. 
Carson, D. C., \& Vecchio, J. M. (2015). Leaving the gang: A review and thoughts on future research. In S. H. Decker \& D. C. Pyrooz (Eds.), The handbook of gangs (pp.257-275). New York: John Wiley \& Sons, Inc.

Clark, C. (1987). Sympathy biography and sympathy margin. American Journal of Sociology, $93,290-321$

Cloward, R. A., \& Ohlin, L. E. (1960). Delinquency and opportunity: A theory of delinquent gangs. Glencoe, Ill: Free Press.

Cohen, A. K. (1955). Delinquent boys: The culture of the gang. Glencoe, Ill: Free Press.

Colvin, M., Cullen, F. T., \& Vander Ven, T. (2002). Coercion, social support, and crime: An emerging theoretical consensus. Criminology, 40, 19-42.

Cullen, F. T. (1994). Social support as an organizing concept for criminology: Presidential address to the Academy of Criminal Justice Sciences. Justice Quarterly, 11, 527-559.

Curry, G. D. (2000). Self-reported gang involvement and officially recorded delinquency. Criminology, 38, 1253-1274.

Dalal, D., \& Zickar, M. J. (2012). Some common myths about centering predictor variables in moderated multiple regression and polynomial regression. Organizational Research Methods, 15, 339-362.

Decker, S. H., \& Curry, G. D. (2000). Addressing key features of gang membership: Measuring the involvement of young members. Journal of Criminal Justice, 28, 473-482.

Decker, S. H., \& Lauritsen, J. (2002). Leaving the gang. In C. R. Huff(Ed.), Gangs in American (3rd ed., pp. 51-70). Thousand Oaks, CA: Sage.

Decker, S. H., \& Van Winkle, B. (1996). Life in the gang: Family, friends and violence. Cambridge, England: Cambridge University Press. 
Decker, S.H., Pyrooz, D. C., \& Moule, R. K. (2014). Disengagement from gangs as role transitions. Journal of Research on Adolescence, 24, 268-283.

Decker, S. H., Pyrooz, D. C., Sweeten, G., Moule, R. K. (2014). Validating self-nomination in gang research: Assessing differences in gang embeddedness across non-, current, and former gang members. Journal of Quantitative Criminology, 30, 577-598.

Dong, B., Gibson, C. L., \& Krohn, M. D. (2015). Gang membership in a developmental and life course perspective. In S. H. Decker \& D. C. Pyrooz (Eds.), The handbook of gangs (pp. 78-97). New York: John Wiley \& Sons, Inc.

Dong, B. \& Krohn, M. D. (2016). Dual trajectories of gang affiliation and delinquent peer association during adolescence: An examination of long-term offending outcomes. Journal of Youth and Adolescence, 45, 746-762.

Dorius, C. J., Bahr, S. J., Hoffmann, J. P., \& Harmon, E. L. (2004). Parenting practices as moderators of the relationship between peers and adolescent marijuana use. Journal of Marriage and Family, 66, 163-178.

Elder, G. H. (1998). The life course as developmental theory. Child Development, 69, 1-12.

Esbensen, F. A., \& Deschenes, E. P. (1998). A multisite examination of youth gang membership: Does gender matter? Criminology, 36, 799-827.

Esbensen, F. A., Winfree, L. T., He, N., \& Taylor, T. J. (2001). Youth gangs and definitional Issues: When is a gang a gang, and why does it matter? Crime \& Delinquency, 47, 105130.

Frauenglass, S., Routh, D. K., Pantin, H. M., \& Mason, C. A. (1997). Family support decreases influence of deviant peers on Hispanic adolescents' substance use. Journal of Clinical Child Psychology, 26, 15-23. 
Gilman, A. B., Hill, K. G., \& Hawkins, J. D. (2014). Long-term consequences of adolescent gang membership for adult functioning. American Journal of Public Health, 104, 938945.

Giordano, P. C., Schroeder, R. D., \& Cernkovich, S. A. (2007). Emotions and crime over the life course: A neo-Meadian perspective on criminal continuity and change. American Journal of Sociology, 112, 1603-1661.

Hagan, J., \& McCarthy, B. (1997). Mean streets: Youth crime and homelessness. New York: Cambridge University Press.

Hagedorn, J. M. (1998). Gang violence in the postindustrial era. Crime and Justice, 24, 365-419. Hennigan, K., \& Spanovic, M. (2012). Gang dynamics through the lens of social identity theory. In F. Esbensen \& C. L. Maxson (Eds.), Youth gangs in international perspective (pp.127149). New York: Springer.

Hipp, J. R., \& Bauer, D. J. (2006). Local solutions in the estimation of growth mixture models. Psychological Methods, 11, 36-53.

Howell, J. C. (2012). Gangs in America's communities. Thousand Oaks, Calif.: SAGE Publications.

Huizinga, D. (1996). The influence of delinquent peers, gangs, and co-offending on violence. Washington, DC: Official of Juvenile Justice and Delinquency Prevention.

Klein, M. W. (1971). Street gangs and street workers. Englewood Cliffs, N.J.: Prentice-Hall. Klein, M. W. (1996). Gangs in the United States and Europe. European Journal on Criminal Policy and Research, 4, 63-80.

Klein, M. W., \& Maxson, C. L. (2006). Street gang patterns and policies. New York: Oxford University Press. 
Krohn, M. D., \& Thornberry, T. P. (2008). Longitudinal perspectives on adolescent street gangs. In A. Liberman (Ed.), The long view of crime: A synthesis of longitudinal research (pp. 128-160). New York: Springer.

Krohn, M. D., Ward, J. T., Thornberry, T. P., Lizotte, A. J., \& Chu, R. (2011). The cascading effects of adolescent gang involvement across the life course. Criminology, 49, 991-1028.

Lacourse, E., Nagin, D., Tremblay, R. E., Vitaro, F., \& Claes, M. (2003). Developmental trajectories of boys' delinquent group membership and facilitation of violent behaviors during adolescence. Development and Psychopathology, 15, 183-197.

Laub, J. H., \& Sampson, R. J. (2003). Shared beginnings, divergent lives: Delinquent boys to age 70. Cambridge, Mass.: Harvard University Press.

Levitt, S. D., \& Venkatesh, S. A. (2001a). An analysis of the long-run consequences of gang involvement. Paper presented at the 2001 Harvard Inequality Summer Institute, Harvard University, Cambridge, MA.

Levitt, S. D., \& Venkatesh, S. A. (2001b). Growing up in the projects: The economic lives of a cohort of men who came of age in Chicago public housing. The American Economic Review, 91, 79-84.

Lin, N. (1986). Conceptualizing social support. In N. Lin, A. Dean, \& W. Ensel (Eds.), Social support, life events, and depression (pp. 17-30). Orlando: Academic Press.

Losel, F., \& Farrington, D. P. (2012). Direct protective and buffering protective factors in the development of youth violence. American Journal of Preventive Medicine, 43, S8-S23.

Marshal, M. P., \& Chassin, L. (2000). Peer influence on adolescent alcohol use: The moderating role of parental support and discipline. Applied Development Science, 4, 80-88. 
Maruna, S. (2001). Making good: How ex-convicts reform and rebuild their lives. Washington, D.C.: American Psychological Association Books

Maxson, C. L. (2013). Do not shoot the messenger: The utility of gang risk research in program targeting and content. Criminology \& Public Policy, 12, 421-426.

McLanahan, S. (2009). Fragile families and the reproduction of poverty. The Annals of the American Academy of Political and Social Sciences, 621, 111-131.

Melde, C., \& Esbensen, F. A. (2011). Gang membership as a turning point in the life course. Criminology, 49, 513-552.

Melde, C., \& Esbensen, F. A. (2014). The relative impact of gang status transitions: Identifying the mechanisms of change in delinquency. Journal of Research in Crime and Delinquency, 51, 349-376.

Miller, J. (2001). One of the guys: Girls, gangs and gender. New York: Oxford University Press.

Miller, W. B. (1958). Lower class culture as a generating milieu of gang delinquency. Journal of Social Issues, 14, 5-19.

Moffitt, T. E. (1993). Adolescence-limited and life-course-persistent antisocial behavior: A developmental taxonomy. Psychological Review, 100, 674-701.

Moloney, M., MacKenzie, K., Hunt, G., \& Joe-Laidler, K. (2009). The path and promise of fatherhood for gang members. British Journal of Criminology, 49, 305-325.

Moore, J. W. (1991). Going down to the barrio: Homeboys and homegirls in change. Philadelphia: Temple University Press.

Moore, J. W., \& Vigil, J. D. (1989). Chicano gangs: Group norms and individual factors related to adult criminality. Aztlan, 18, 31-42 
Nagin, D. (2005). Group-based modeling of development. Cambridge, Mass: Harvard University Press.

Osgood, D. W., \& Lee, H. (1993). Leisure activities, age, and adult roles across the lifespan. Society and Leisure, 16, 181-208.

Papachristos, A. V. (2009). Murder by structure: Dominance relations and the social structure of gang homicide. American Journal of Sociology, 115, 74-128.

Poole, E. D., \& Regoli, R. M. (1979). Parental support, delinquent friends, and delinquency: A test of interaction effects. Journal of Criminal Law and Criminology, 70, 188-193.

Pyrooz, D. C. (2013). Gangs, criminal offending, and an inconvenient truth. Criminology \& Public Policy, 12, 427-436.

Pyrooz, D. C. (2014). From colors and guns to caps and gowns? The effects of gang membership on educational attainment. Journal of Research in Crime and Delinquency, 51, 56-87.

Pyrooz, D. C., \& Decker, S. H. (2011). Motives and methods of leaving the gang: Understanding the process of gang desistance. Journal of Criminal Justice, 39, 417-425.

Pyrooz, D. C., Decker, S. H., \& Webb, V. J. (2014). The ties that bind: Desistance from gangs. Crime \& Delinquency, 60, 491-516.

Pyrooz, D. C., \& Sweeten, G. (2015). Gang membership between ages 5 and 17 years in the United States. Journal of Adolescent Health, 56, 414-419.

Pyrooz, D. C., Sweeten, G., \& Piquero, A. R. (2013). Continuity and change in gang membership and gang embeddedness. Journal of Research in Crime and Delinquency, 50, 239-271.

Raudenbush, S. W. (2005). How do we study what happen next? The Annals of the American Academy of Political and Social Science, 602, 131-144. 
Rosenberg, M. (1965). Society and the adolescent self-image. Princeton, N.J.: Princeton University Press.

Rosenberg, M., \& McCullough, C. B. (1981). Mattering: Inferred significance and mental health among adolescents. Research in Community and Mental Health, 2, 163-182.

Sampson, R. J., \& Laub, J. H. (1993). Crime in the making: Pathways and turning points through life. Cambridge, MA: Harvard University Press.

Sampson, R. J., \& Laub, J. H. (2005). A life-course view of the development of crime. The ANNALS of the American Academy of Political and Social Science, 602, 12-45.

Sampson, R. J, Laub, J. H. \& Wimer, C. (2006). Does marriage reduce crime? A counterfactual approach to within-individual causal effects. Criminology, 44, 465-508.

Sánchez-Jankowski, M. S. (1991). Islands in the street: Gangs and American urban society. Berkeley: University of California Press.

Sharkey, J. D., Stifel, S. W., \& Mayworm, A. M. (2015). How to help me get out of a gang: Youth recommendations to family, school, community, and law enforcement systems. OJJDP Journal of Juvenile Justice, 4, 64-83.

Sherman, L. W. (1993). Defiance, deterrence, and irrelevance: A theory of the criminal sanction. Journal of Research in Crime and Delinquency, 30, 445-473.

Shott, S. (1979). Emotion and social life: A symbolic interactionist analysis. American Journal of Sociology, 84, 1317-334.

Shute, J. (2013). Family support as a gang reduction measure. Children \& Society, 27, 48-59.

Simon, T., Ritter, N., \& Mahendra, R. (2013). Changing course: Preventing gang membership. Washington, D.C.:U.S. Department of Justice, Office of Justice Programs, and the Centers for Disease Control and Prevention. 
Sullivan, M. L. (1989). Getting paid: Youth crime and work in the inner city. Ithaca: Cornell University Press.

Sweeten, G. (2012). Scaling criminal offending. Journal of Quantitative Criminology, 28, 533557.

Taylor, T. J., Freng, A., Esbensen, F., \& Peterson, D. (2008). Youth gang membership and serious violent victimization: The importance of lifestyles and routine activities. Journal of Interpersonal Violence, 23, 1441-1464.

Taylor, T. J., Peterson, D., Esbensen, F., \& Freng, A., (2007). Gang membership as a risk factor for adolescent violent victimization. Journal of Research in Crime and Delinquency, 44, 351-380.

Thoits, P. A. (2011). Mechanisms linking social ties and support to physical and mental health. Journal of Health and Social Behavior, 52, 145-161.

Thornberry, T. P., Krohn, M. D., Lizotte, A. J., \& Chard-Wierschem, D. (1993). The role of juvenile gangs in facilitating delinquent behavior. Journal of Research in Crime and Delinquency, 30, 55-87.

Thornberry, T. P., Krohn, M. D., Lizotte, A. J., Smith, C. A., \& Tobin, K. (2003). Gangs and delinquency in developmental perspective. New York, NY: Cambridge University Press.

Uchino, B. N. (2009). Understanding the links between social support and physical health: A life-span perspective with emphasis on the separability of perceived and received Support. Perspectives on Psychological Science, 4, 236-55.

Vigil, J. D. (1988). Barrio gangs: Street life and identity in Southern California. Austin: University of Texas Press. 
Vitaro, F., Brendgen, M., \& Tremblay, R. E. (2000). Influence of deviant friends on delinquency: Searching for moderator variables. Journal of Abnormal Child Psychology, 28, 313-325.

Walker-Barnes, C. J., \& Mason, C. A. (2004). Delinquency and substance use among ganginvolved youth: The moderating role of parenting practices. American Journal of Community Psychology, 34, 235-250.

Warr, M. (1998). Life-course transitions and desistance from crime. Criminology, 36, 183-216.

Wood, J., Foy, D. W., Layne, C., Pynoos, R., \& James, C. B. (2002). An examination of the relationships between violence exposure, posttraumatic stress symptomatology, and delinquent activity: An "ecopathological" model of delinquent behavior among incarcerated adolescents. Journal of Aggression, Maltreatment, and Trauma, 6, 127-147.

Wright, J. P., \& Cullen, F. T. (2001). Parental efficacy and delinquent behavior: Do control and support matter? Criminology, 39, 677-706. 


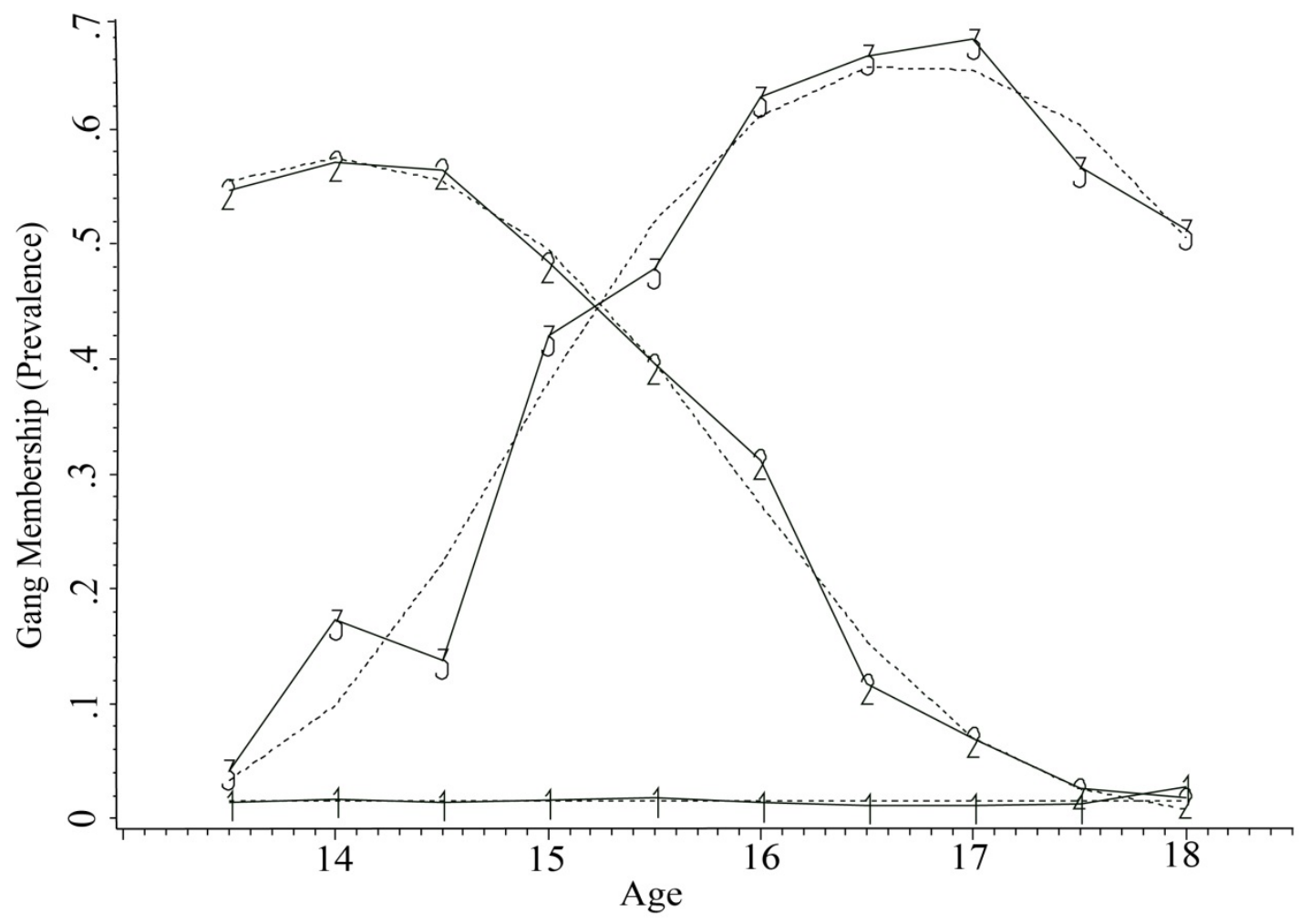

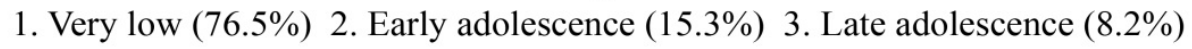

Figure 1. Developmental trajectories of gang affiliation during adolescence 
Table 1. Descriptive statistics $(N=560)$

\begin{tabular}{lccccc}
\hline Variables & $\begin{array}{c}\text { Mean or } \\
\text { proportion }\end{array}$ & S.D. & Min & Max & Wave \\
\hline Violence & 0.136 & 0.387 & 0.000 & 2.000 & $13-14$ \\
GM1 & 0.774 & 0.370 & 0.000 & 0.996 & $2-9$ \\
GM2 & 0.144 & 0.282 & 0.000 & 0.999 & $2-9$ \\
GM3 & 0.083 & 0.243 & 0.000 & 1.000 & $2-9$ \\
Consistency of parental support & 0.000 & 1.000 & -1.140 & 1.456 & $10-12$ \\
Consistency of parental attachment & 0.000 & 1.000 & -1.119 & 1.460 & $10-12$ \\
Partner status (Wave 10) & 0.505 & 0.500 & 0.000 & 1.000 & 10 \\
African American & 0.629 & 0.484 & 0.000 & 1.000 & 1 \\
Hispanic & 0.175 & 0.380 & 0.000 & 1.000 & 1 \\
Parental education & 11.539 & 2.190 & 6.000 & 18.000 & 1 \\
Family poverty & 0.276 & 0.448 & 0.000 & 1.000 & 2 \\
Parental supervision & 3.595 & 0.407 & 1.500 & 4.000 & 2 \\
Self esteem & 3.079 & 0.405 & 1.778 & 4.000 & 2 \\
Depression & 2.106 & 0.436 & 1.000 & 3.571 & 2 \\
Prior violence (Wave 2) & 0.585 & 0.928 & 0.000 & 4.000 & 2 \\
Aggression & 0.461 & 0.355 & 0.000 & 1.833 & 3 \\
Academic Aptitude & 58.468 & 26.290 & 1.000 & 99.000 & $\mathrm{a}^{*}$ \\
Neighborhood disadvantage & -0.059 & 0.987 & -2.262 & 1.511 & $\mathrm{~b}^{*}$ \\
\hline Note: GM-gang ma & & & & \\
\hline
\end{tabular}

Note: GM=gang membership trajectory group; a: measured by the math percentile score received on the California Achievement Test in 1987 (prior to Wave 1 of the RYDS); b: measured by 1990 U.S. Census. 
Table 2. Poisson regression of violence on gang affiliation trajectories $(N=560)$

\begin{tabular}{lcc}
\hline Variables & i.r.r (s.e.) & p-value \\
\hline GM2 & $2.587(0.888)$ & $0.006^{*}$ \\
GM3 & $2.986(1.217)$ & $0.007^{*}$ \\
African American & $1.109(0.447)$ & 0.798 \\
Hispanic & $0.953(0.494)$ & 0.926 \\
Neighborhood disadvantage & $1.171(0.178)$ & 0.299 \\
Parental education & $1.070(0.063)$ & 0.247 \\
Family poverty & $1.033(0.325)$ & 0.918 \\
Parental supervision & $0.979(0.289)$ & 0.941 \\
Self esteem & $1.019(0.364)$ & 0.958 \\
Depression & $1.003(0.321)$ & 0.993 \\
Academic Aptitude & $1.009(0.005)$ & $0.085^{\wedge}$ \\
Aggression & $1.805(0.610)$ & $0.080^{\wedge}$ \\
Prior violence (Wave 2) & $1.147(0.155)$ & 0.309
\end{tabular}

Note: $\mathrm{GM}=$ gang membership trajectory group; i.r.r. $=$ incidence rate ratio; s.e. $=$ standard error ${ }^{\wedge} \mathrm{p}<0.10 ;{ }^{*} \mathrm{p}<0.05$ 
Table 3. Poisson regression of violence on gang affiliation trajectories, partner status (Wave 10) and their interactions $(N=560)$

\begin{tabular}{|c|c|c|c|c|}
\hline \multirow[b]{2}{*}{ Variables } & \multicolumn{2}{|c|}{ Model 1} & \multicolumn{2}{|c|}{ Model 2} \\
\hline & i.r.r. (s.e.) & p-value & i.r.r (s.e.) & p-value \\
\hline GM2 & $2.334(0.817)$ & $0.015^{*}$ & $6.496(3.096)$ & $<0.001 *$ \\
\hline GM3 & $2.622(1.063)$ & $0.017 *$ & $3.024(2.206)$ & $0.099^{\wedge}$ \\
\hline Partner status (Wave 10) & $1.677(0.420)$ & $0.039^{*}$ & $2.611(0.860)$ & $0.004 *$ \\
\hline Interaction: GM2*partner status & -- & -- & $0.194(0.130)$ & $0.015^{*}$ \\
\hline Interaction: GM3*partner status & -- & -- & $0.794(0.605)$ & 0.762 \\
\hline African American & $1.086(0.434)$ & 0.836 & $1.040(0.394)$ & 0.917 \\
\hline Hispanic & $0.878(0.453)$ & 0.801 & $0.879(0.432)$ & 0.793 \\
\hline Neighborhood disadvantage & $1.183(0.180)$ & 0.268 & $1.199(0.178)$ & 0.222 \\
\hline Parental education & $1.080(0.066)$ & 0.205 & $1.075(0.064)$ & 0.225 \\
\hline Family poverty & $0.994(0.311)$ & 0.986 & $0.964(0.295)$ & 0.905 \\
\hline Parental supervision & $0.990(0.294)$ & 0.974 & $0.958(0.291)$ & 0.888 \\
\hline Self esteem & $0.972(0.341)$ & 0.936 & $0.989(0.365)$ & 0.976 \\
\hline Depression & $1.017(0.315)$ & 0.956 & $0.994(0.314)$ & 0.984 \\
\hline Academic Aptitude & $1.009(0.005)$ & $0.083^{\wedge}$ & $1.009(0.005)$ & $0.073^{\wedge}$ \\
\hline Aggression & $1.757(0.591)$ & $0.094^{\wedge}$ & $1.880(0.632)$ & $0.060^{\wedge}$ \\
\hline Prior violence (Wave 2) & $1.167(0.154)$ & 0.242 & $1.145(0.152)$ & 0.308 \\
\hline
\end{tabular}

Note: $\mathrm{GM}=$ gang membership trajectory group; i.r.r.=incidence rate ratio; s.e. $=$ standard error

${ }^{\wedge} \mathrm{p}<0.10 ;{ }^{*} \mathrm{p}<0.05$ 
Table 4. Poisson regression of violence on gang affiliation trajectories, parental support and attachment, and their interactions $(N=560)$

\begin{tabular}{|c|c|c|c|c|c|c|}
\hline \multirow[b]{2}{*}{ Variables } & \multicolumn{2}{|c|}{ Model 1} & \multicolumn{2}{|c|}{ Model 2} & \multicolumn{2}{|c|}{ Model 3} \\
\hline & i.r.r. (s.e.) & p-value & i.r.r (s.e.) & p-value & i.r.r.(s.e.) & p-value \\
\hline GM2 & $2.498(0.880)$ & $0.009 *$ & $2.415(0.892)$ & $0.017 *$ & $2.972(1.072)$ & $0.003 *$ \\
\hline GM3 & $3.055(1.189)$ & $0.004 *$ & $2.121(0.900)$ & $0.076^{\wedge}$ & $2.448(1.039)$ & $0.035^{*}$ \\
\hline Consistency of parental support & $0.944(0.130)$ & 0.674 & $1.025(0.165)$ & 0.876 & $0.957(0.132)$ & 0.748 \\
\hline Consistency of parental attachment & $0.695(0.105)$ & $0.016^{*}$ & $0.716(0.110)$ & $0.029 *$ & $0.643(0.136)$ & $0.037 *$ \\
\hline Interaction: GM2* parental support & -- & -- & $1.056(0.354)$ & 0.872 & -- & -- \\
\hline Interaction: GM3*parental support & -- & -- & $0.361(0.153)$ & $0.016^{*}$ & -- & -- \\
\hline Interaction: GM2*parental attachment & -- & -- & -- & -- & $1.658(0.595)$ & 0.159 \\
\hline Interaction: GM3*parental attachment & -- & -- & -- & -- & $0.629(0.239)$ & 0.223 \\
\hline African American & $1.088(0.434)$ & 0.832 & $1.054(0.423)$ & 0.895 & $1.055(0.420)$ & 0.892 \\
\hline Hispanic & $0.915(0.465)$ & 0.861 & $0.864(0.427)$ & 0.767 & $0.903(0.450)$ & 0.838 \\
\hline Neighborhood disadvantage & $1.151(0.171)$ & 0.343 & $1.154(0.177)$ & 0.348 & $1.154(0.173)$ & 0.339 \\
\hline Parental education & $1.062(0.062)$ & 0.303 & $1.077(0.063)$ & 0.205 & $1.062(0.060)$ & 0.282 \\
\hline Family poverty & $1.034(0.320)$ & 0.915 & $0.958(0.307)$ & 0.893 & $1.031(0.326)$ & 0.923 \\
\hline Parental supervision & $1.101(0.324)$ & 0.744 & $1.200(0.353)$ & 0.537 & $1.100(0.334)$ & 0.752 \\
\hline Self esteem & $1.121(0.390)$ & 0.743 & $1.026(0.376)$ & 0.945 & $1.122(0.387)$ & 0.738 \\
\hline Depression & $0.933(0.298)$ & 0.829 & $0.915(0.283)$ & 0.775 & $0.941(0.300)$ & 0.847 \\
\hline Academic Aptitude & $1.007(0.005)$ & 0.177 & $1.006(0.005)$ & 0.208 & $1.006(0.005)$ & 0.231 \\
\hline Aggression & $1.587(0.559)$ & 0.190 & $1.614(0.579)$ & 0.182 & $1.615(0.554)$ & 0.163 \\
\hline Prior violence (Wave 2) & $1.177(0.157)$ & 0.223 & $1.240(0.162)$ & 0.101 & $1.160(0.156)$ & 0.271 \\
\hline
\end{tabular}

Note: $\mathrm{GM}=$ gang membership trajectory group; i.r.r.=incidence rate ratio; s.e. $=$ standard error $\wedge \mathrm{p}<0.10 ;{ }^{*} \mathrm{p}<0.05$ 